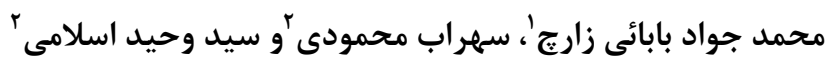

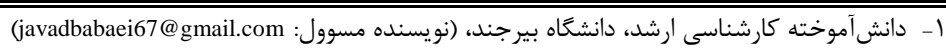

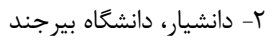

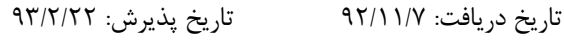

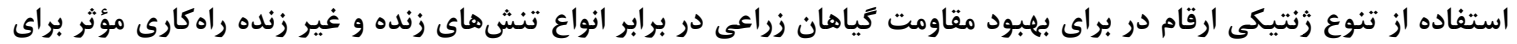

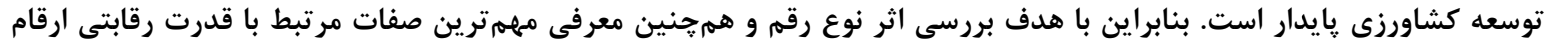

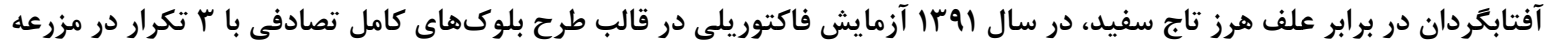

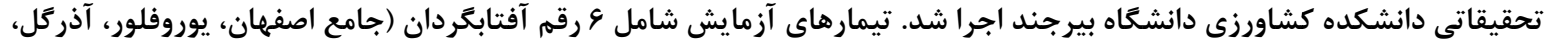

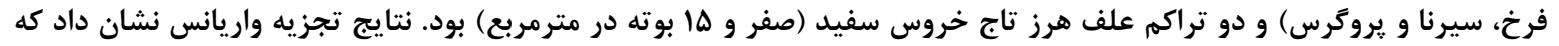

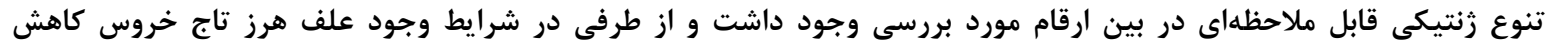

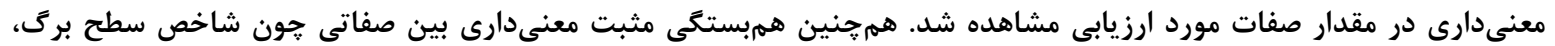

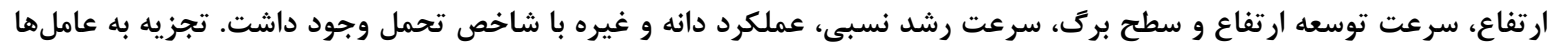

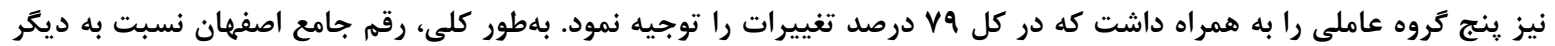

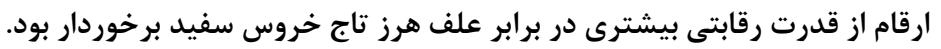

وازههاى كليدى: جامع اصفهان، شاخص تحمل، شاخص سطح برك، قدرت رقابتى، كشاورزى پايدار

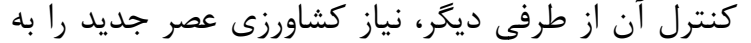

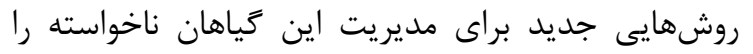

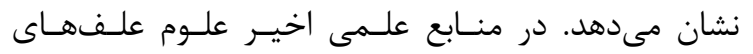

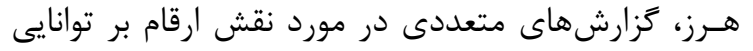

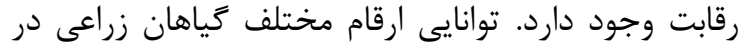

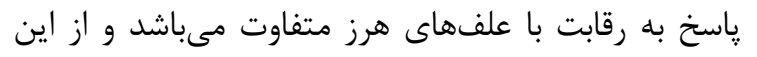

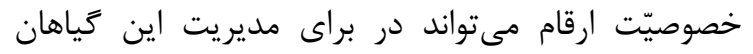

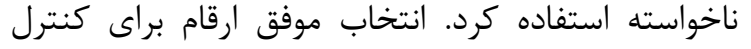

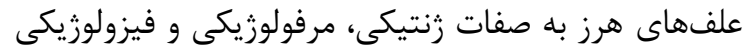

خاص وابسته مى باشد به (1) (1).

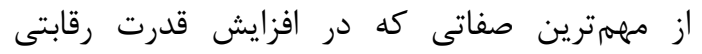

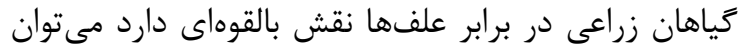

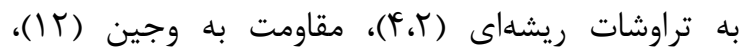

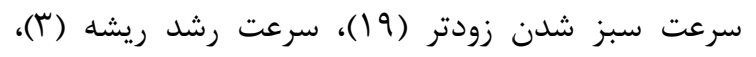

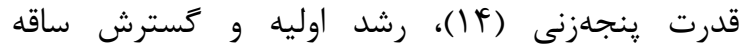

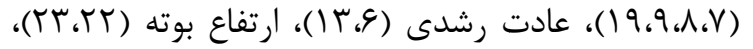

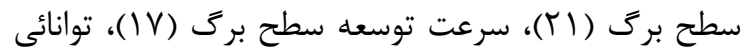

در بين محصولات كشاورزى متنوعى كه در دمايد سطح

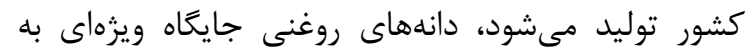

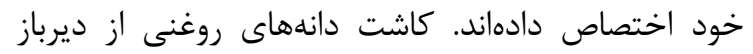

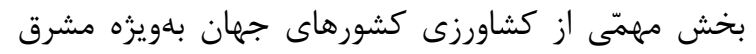

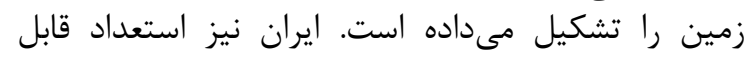

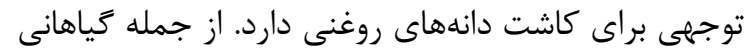

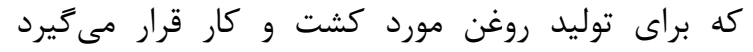

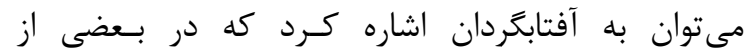

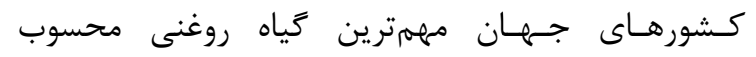
مىشود كه مورد كشتو كار قرار مى تيرد (1) (1).

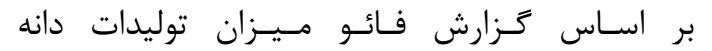

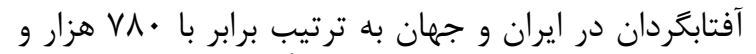

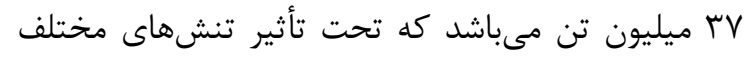

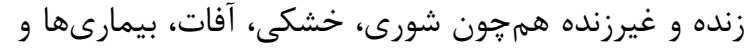

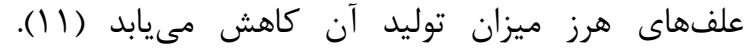
خسارت وجود علفهاى هرز از يك طرف آن و هزئ هزينهائ 


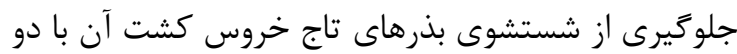

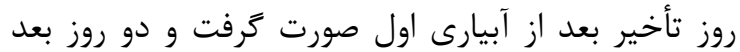

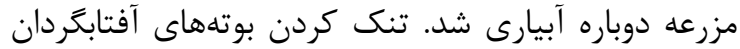

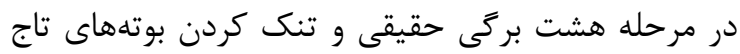

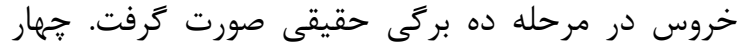

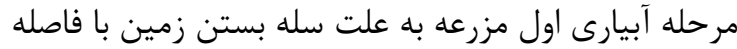

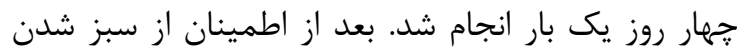

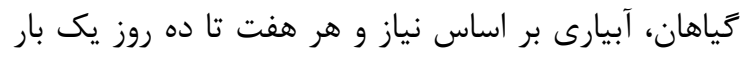

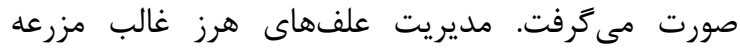

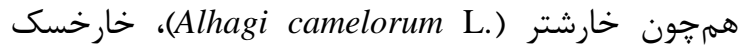
Convolvulus L.) (Tribulus terrestris L.) (arvensis دستى طى سه مرحله صورت كرفت.

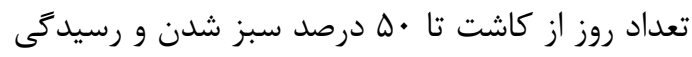

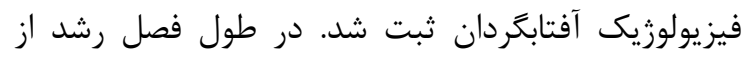

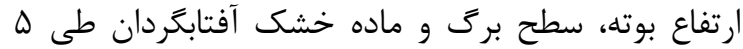

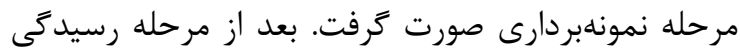

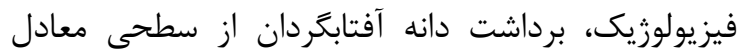

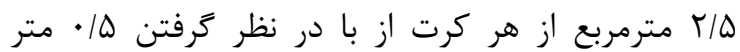

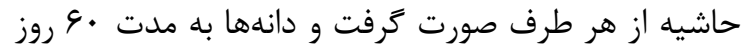

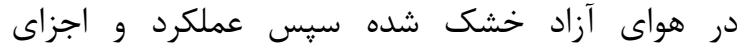

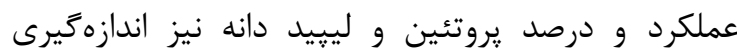

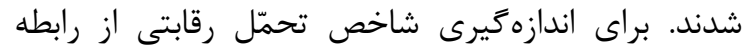

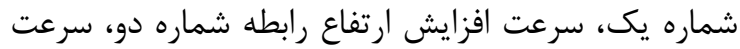

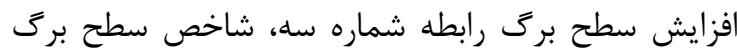

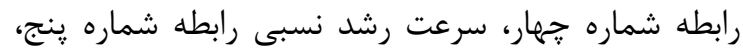

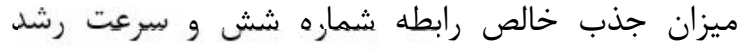
محصول از رابطه شماره هفت استفاده شد. شئر

$$
\begin{aligned}
& A w c=\frac{V \text { infested }}{V \text { pure }} \\
& R \mathrm{HE}=\frac{\mathrm{h} 2-\mathrm{h} 1}{\mathrm{t} 2-\mathrm{t} 1} \\
& R L A E=\frac{L A 2-L A I}{t 2-t 1} \\
& L A I=\frac{l A 2+l A 1}{2} \times \frac{1}{G A} \\
& R G R=\frac{\ln W 2-\ln W 1}{T 2-T 1} \\
& C G R=\frac{1}{G A} \cdot \frac{W_{2}-W 1}{T 2-T 1} \\
& N A R=\frac{W 2-W 1}{T 2-T 1} \cdot \frac{\ln L A 2-\ln L A 1}{L A 2-L A 1}
\end{aligned}
$$

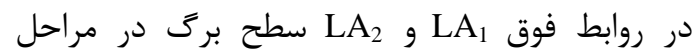

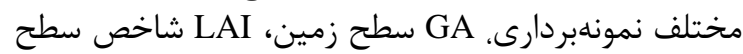

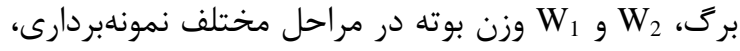
RGR نشان سرعت رشد نسبى، CGR سرعت رخد رشد محصول، NAR

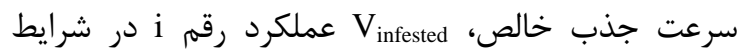

حفظ عملكرد (·r) و و توانايى كاهش زيست توده علف هرز

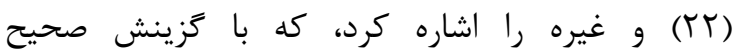

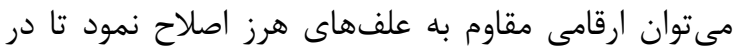

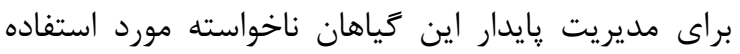

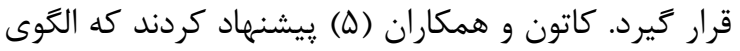

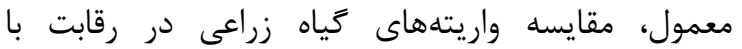

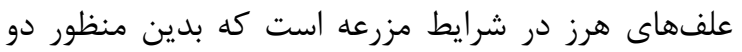

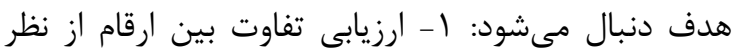

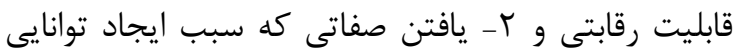

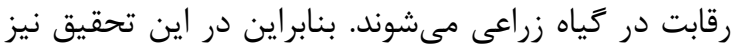

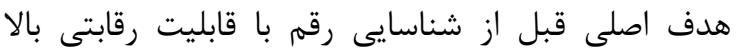

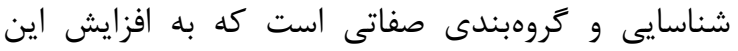

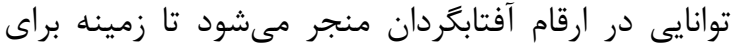

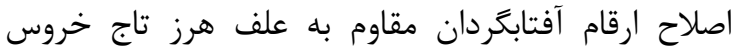
سفيد مهيا كردد.

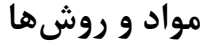

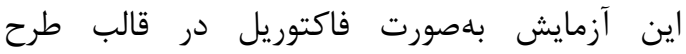

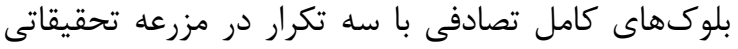

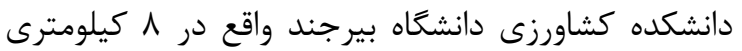

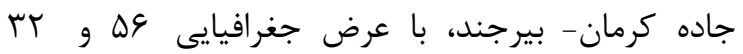

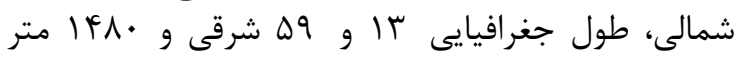

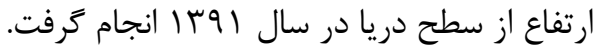

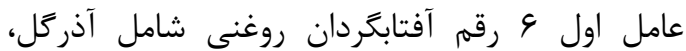

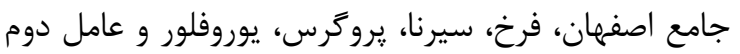

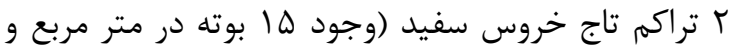

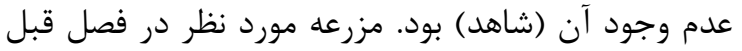

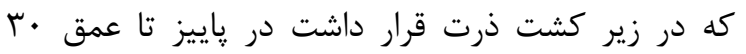

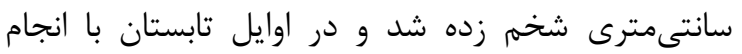

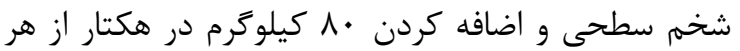

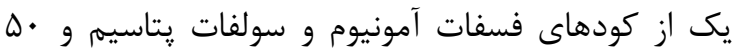

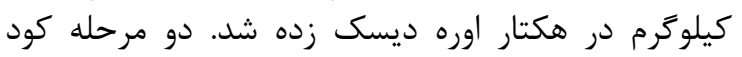

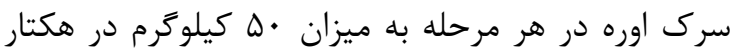

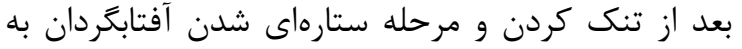

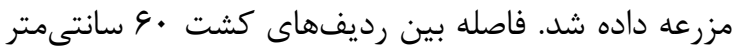

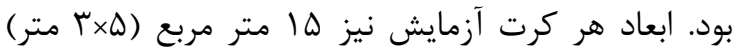

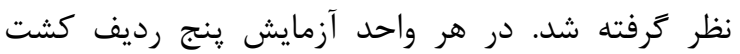

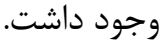

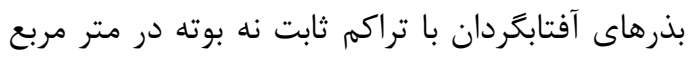

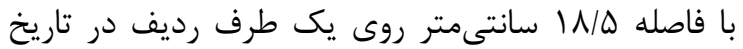

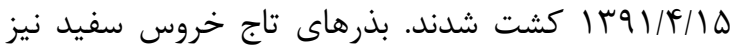

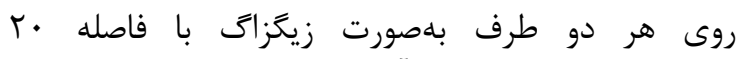

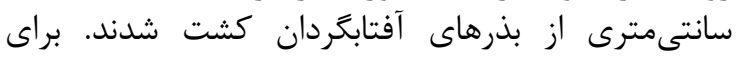




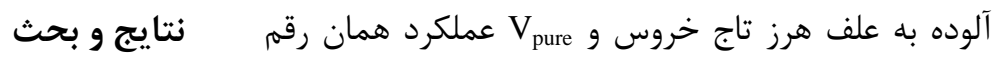

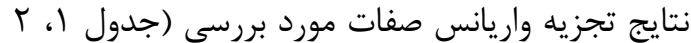

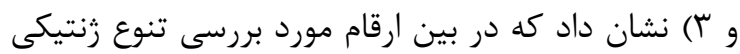

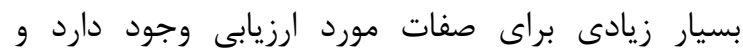

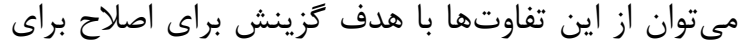
اهداف مورد نظر مورد استفاده قرار كيرد.

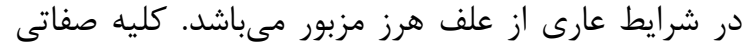

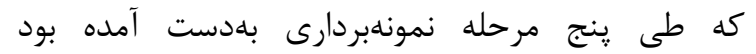

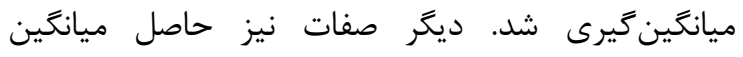

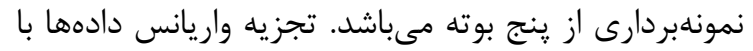

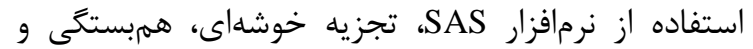

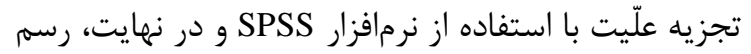

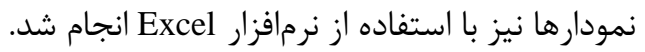

\begin{tabular}{|c|c|c|c|c|c|c|c|c|}
\hline \multicolumn{7}{|c|}{ ميانگين مربعات } & \multirow[b]{2}{*}{ آزادى } & \multirow[b]{2}{*}{ منابع تغيير } \\
\hline سرعت رشد & سرعت رشد & سرعت جذب & سرعت افزايش & شاخص سطح & فيزيولوزيكى & سبز شدن & & \\
\hline $4 / \wedge 9^{* 3}$ & $\Lambda / \Lambda \Delta^{*}$ & $1 / V \cdot$ & $19 \Delta / \Delta 9$ & $\cdot / V r^{N *}$ & $T r / \cdot T^{*}$ & $19 / \mathrm{VV}^{*}$ & r & بلوك \\
\hline$\cdot / \cdots r^{* \pi}$ & $\mathrm{V} / \cdot \mathrm{V}^{\mathrm{*}}$ & $9 / 94$ & rrq/q६ & $\cdot / \pi \Lambda^{\pi}$ & $\Lambda F / \|\left.\right|^{* \pi}$ & $19 / 4 f^{* \pi}$ & $\Delta$ & رقم (C) \\
\hline$\cdot / \cdots q^{m *}$ & $\Pi \backslash \Lambda / \Delta F^{n-m}$ & $194 / 1 r^{m-m}$ & IFAK/AT & $r / \Lambda f^{n-m}$ & $4 q^{* m}$ & i & 1 & علف هرز (D) \\
\hline$\cdot 1 \cdots \cdot v$ & $T / V I$ & $|\varepsilon / \pi|^{m * n}$ & $\Lambda \cdot / 4 \Lambda$ & $\cdot / 49$ & D/9६ & .1 .99 & $\Delta$ & $\mathrm{C} \times \mathrm{D}$ \\
\hline$\cdot 1 \cdot \cdots \cdot v$ & $r / l$. & G/OV & $q \% / \cdot \Lambda$ &.$/ 14$ & D/G & $1 / \pi 9$ & tr & خطا \\
\hline$r / Q F$ & $I r / V V$ & $1 \pi / 9$ & rr/s9 & $\mid N / F \Lambda$ & T/QT & $1 T / v q$ & - & $\mathrm{CV} \%$ \\
\hline
\end{tabular}

جدول r- تجزيه واريانس صفات مرتبط با ارتفاع، قطر ساقه و عملكرد كمّى و كيفى آفتابكردان

\begin{tabular}{|c|c|c|c|c|c|c|c|c|}
\hline \multicolumn{7}{|c|}{ ميانگين مربعات } & \multirow[b]{2}{*}{ آزادى درجه } & \multirow[b]{2}{*}{ منابع تغيير } \\
\hline عملكرد دانه & عملكرد كلش & يروتئين & عملكرد خربى & قطر ساقه & سرعت افزايش & ارتفاع & & \\
\hline GIIrT. & $191 Y 9 \Lambda$ & THVVQV & 1.9G YTHF" & $\cdot / \cdot r$ & $.1 .94^{\pi}$ & $1 \wedge 9 / V V^{*}$ & r & بلوك \\
\hline 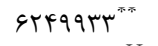 & FAV৭৭IA & VDFTF & DFVTAD & . MFY & $.1 .90^{* \pi}$ & $\Delta \mid \varepsilon / f f^{\pi *}$ & $\Delta$ & رقم (C) \\
\hline 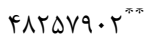 & $\Delta .919 \Delta .^{* \pi}$ & rqpi...* & $11 V \cdot \Delta \Delta q^{*}$ & $\cdot 19 \Delta \cdot{ }^{* \pi}$ & $\cdot / r \mid f^{\pi *}$ & reTV/F & 1 & علف هرز (D) \\
\hline$|\Delta 9 \Delta \cdot F|$ & $519 \cdot 111$ & $r \Delta \cdot \Lambda \Lambda$ & $\Delta \Delta V I T$ & $\cdot|\cdot|+\mid$ & .1 .14 & $1 \cdot 4 / 99^{\pi \pi}$ & $\Delta$ & $\mathrm{C} \times \mathrm{D}$ \\
\hline$V \cdot r \wedge \Delta V$ & $19911 \mathrm{rV}$ & $\varepsilon Y \cdot \Delta V$ & rEDVFF & $\cdot / \cdot r \wedge$ & $.1 \cdot 10$ & rV/AF & tr & خطا \\
\hline $\mid N / T \Lambda$ & TA/FS & TE/TA & TN/q & $\mid r / \cdot T$ & $1.1 \cdot \Delta$ & $\Delta / A \Delta$ & - & CV\% \\
\hline
\end{tabular}

جدول rـ- تجزيه واريانس شاخص تحمل، قطر طبق، شاخص برداشت، درصد يوكى و اجزاى عملكرد دانه آفتابكردان

\begin{tabular}{|c|c|c|c|c|c|c|c|}
\hline \multicolumn{6}{|c|}{ ميانگين مربعات } & \multirow{2}{*}{ درجه آزادى } & \multirow{2}{*}{ منابع تغيير } \\
\hline شاخص تحمل & وزن هزار دانه & درصد يوكى & تعداد دانه در طبق & شاخص برداشت & قطر طبق & & \\
\hline $.1 .919^{\circ}$ & $1 \Delta / V F^{F}$ & $V F / F F$ & TYYDQ & $\cdot / \Delta T^{\prime}$ & $\Delta / 91$ & $r$ & بلوك \\
\hline$\cdot / \cdot v$ & TTt/. r* & $V \wedge G / K \mu^{\pi * *}$ & INTDIV & 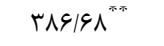 & $r / T r$ & $\Delta$ & رقم (C) \\
\hline $1 / T \wedge r^{* * \pi}$ & TAIT/VT & $f G \mid Y / F \varphi^{*}$ & r. GrGY & • & 1 & 1 & علف هرز (D) \\
\hline$\cdot / \cdot v$ & rIV/qY" & IIT/Ar & TTFDG & $r \cdot 1 / 8)^{*}$ & $r / V I$ & $\Delta$ & $\mathrm{C} \times \mathrm{D}$ \\
\hline .1 .19 & $9 \% / 99$ & $I T \cdot / T V$ & TIFAT & $\Delta \Delta / 99$ & $V / T F$ & tr & خطا \\
\hline $\mid 91 . r$ & IV/TK & ז & $1 \% / \pi$ & $10 / \Gamma q$ & $11 / 49$ & - & CV\% \\
\hline
\end{tabular}

براى شاخصهاى رشدى آفتابكردان نشان داد كه رقابت

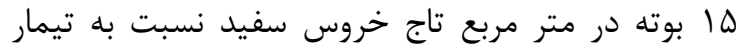

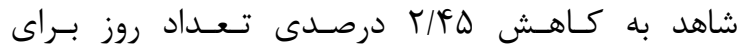

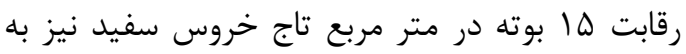

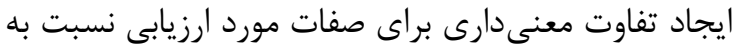

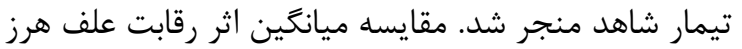




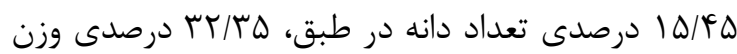

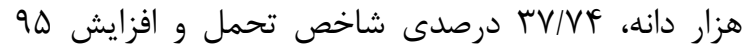

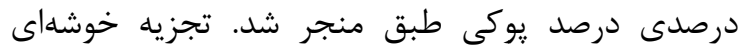

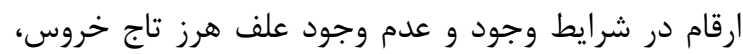

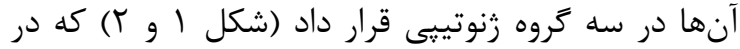

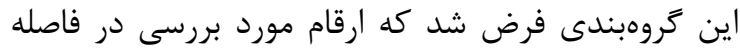

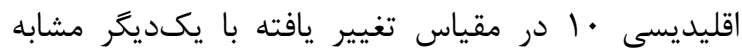

رسيـدگى فيزيولوزيك،

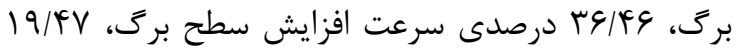
درصدى سرعت جذب خالص، 1 •

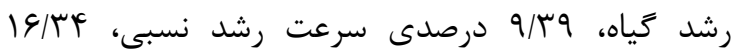

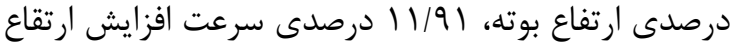

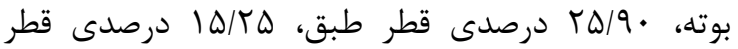
ساقه، • 1N/K درصدى عملكرد روغن،

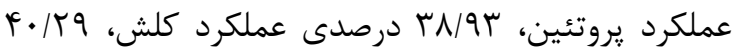

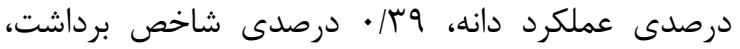
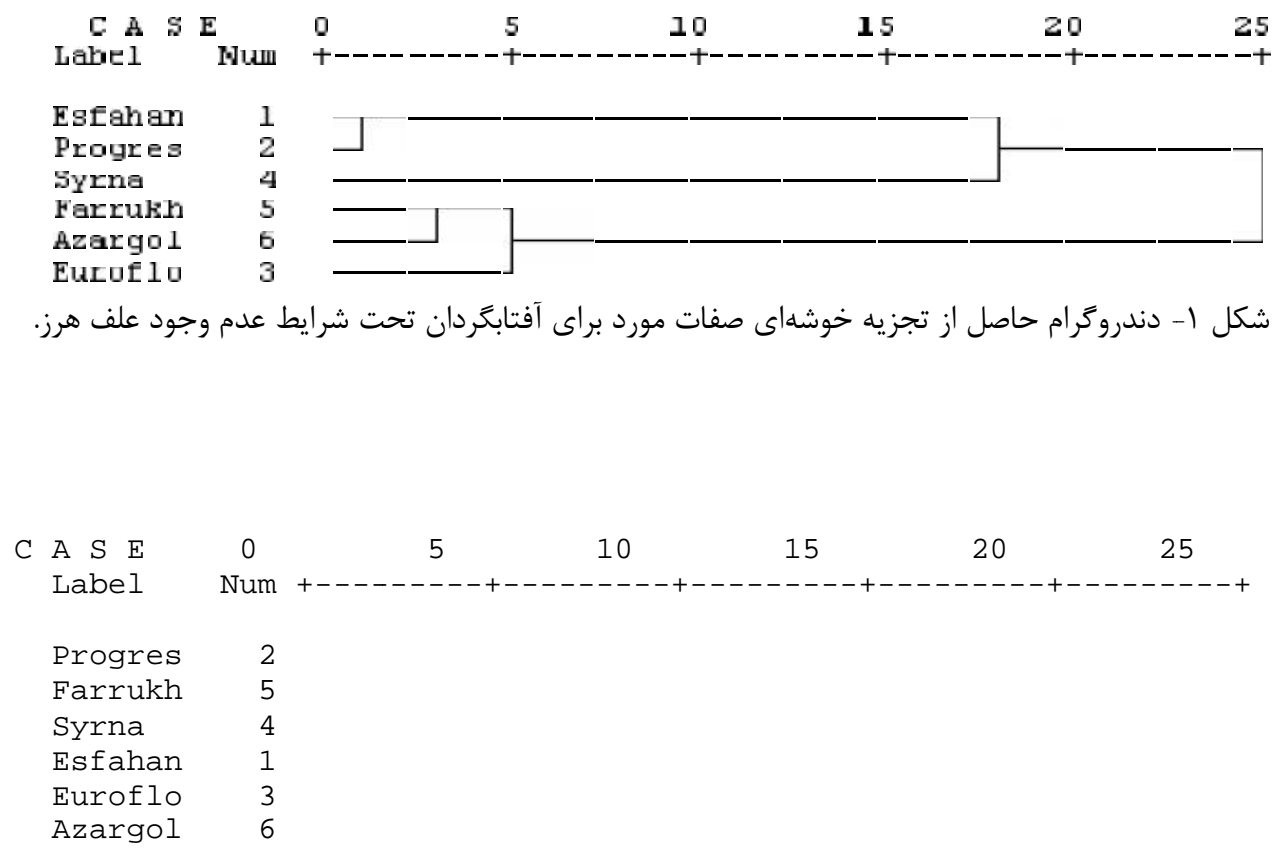

شكل r- دندروگرام حاصل از تجزيه خوشهاى صفات مورد بررسى براى آفتابكردان تحت رقابت با علف هرز.

هزار دانه بود و از لحاظ صفاتى جون سرعت افزايش ارتفاع،

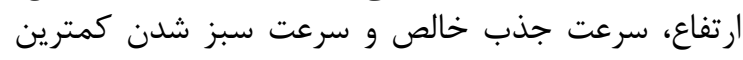
بودند. سرعت سبز شدن هرئ هر جاله

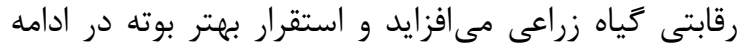

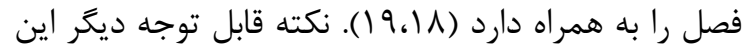

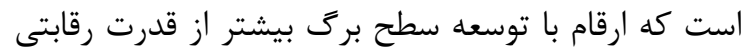

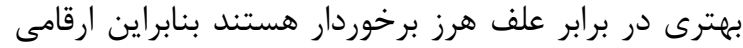

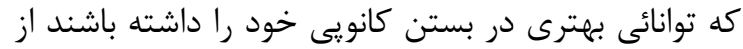

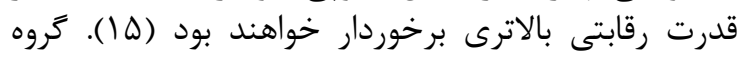

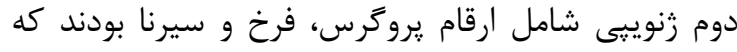

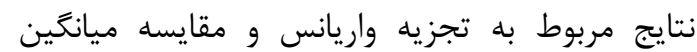

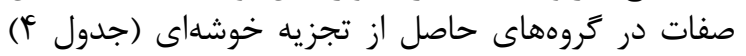

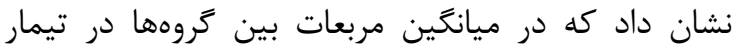

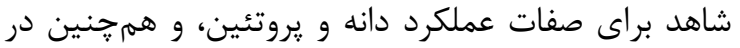

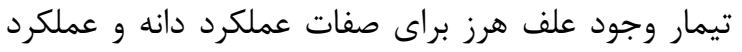

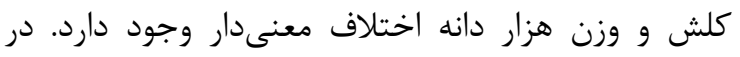

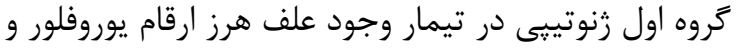

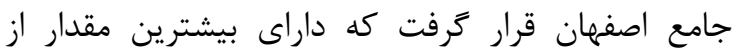

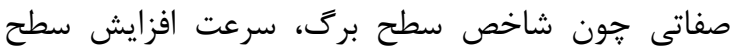

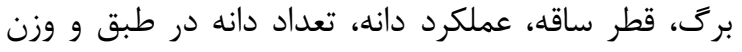




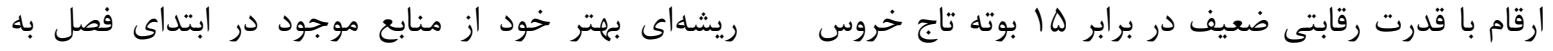

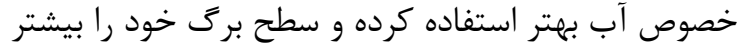

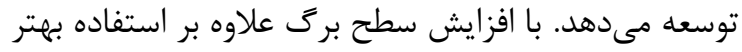

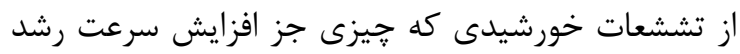

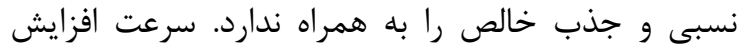

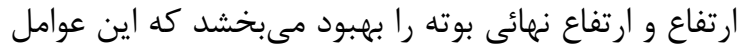

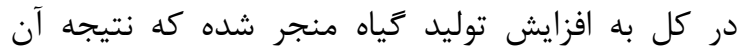

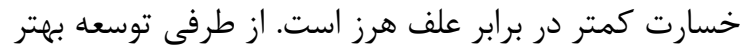

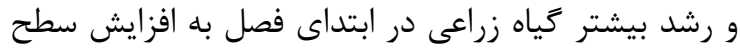

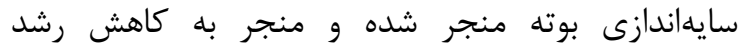

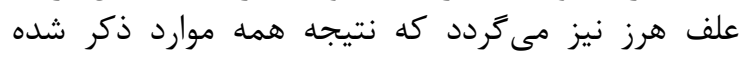

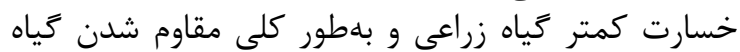

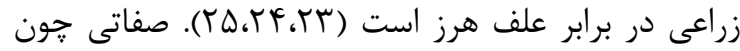

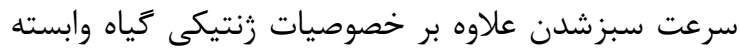

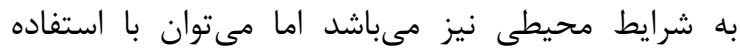

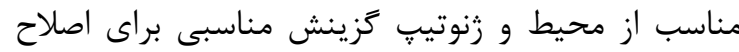

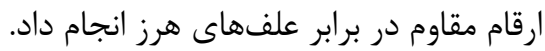

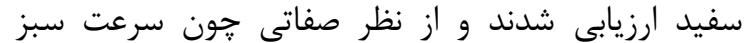

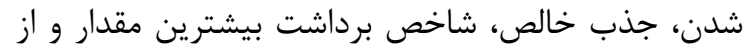

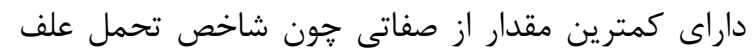

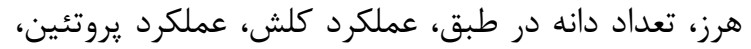

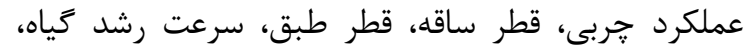

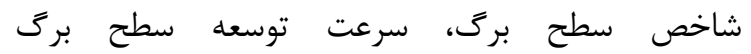

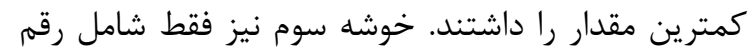

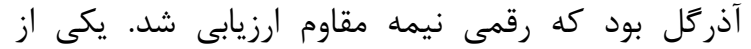

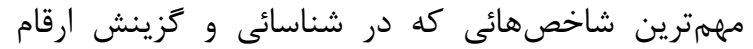

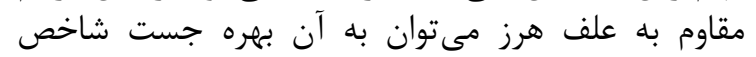

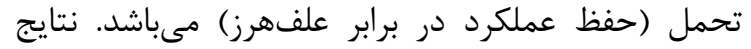

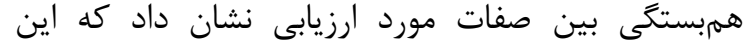

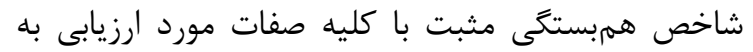

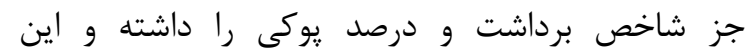

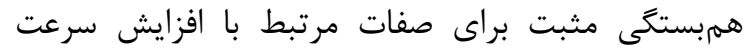

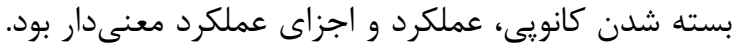
با افزايش سرعت سبز شدن در ابتدا، كياه با توسعه سيسته 
جدول f- نتايج همبستكى صفات مورد مطالعه

\begin{tabular}{|c|c|c|c|c|c|c|c|c|c|c|c|c|c|c|c|c|c|c|c|c|}
\hline$(T \cdot)$ & (19) & $(1 \wedge)$ & (IV) & (19) & $(\mid Q)$ & (If) & (Ir) & $(I T)$ & (1I) & $(1 \cdot)$ & (9) & $(\wedge)$ & (V) & (9) & (b) & (f) & (ए) & (T) & (1) & صفات \\
\hline & & & & & & & & & & & & & & & & & & & 1 & (1) \\
\hline & & & & & & & & & & & & & & & & & & 1 &.$- / T F A$ & (r) \\
\hline & & & & & & & & & & & & & & & & & 1 &. $\mid 411^{\circ}$ &.$- / \cdot 19$ & (r) \\
\hline & & & & & & & & & & & & & & & & 1 &. $\mid g q \psi^{m-m}$ & . /rq4 & $-/ T r \Delta \Delta^{*}$ & $(f)$ \\
\hline & & & & & & & & & & & & & & & 1 & $\cdot 11 \cdot 9$ & $-\cdot / / \gamma$ & - RFA & $-.1 \cdot 9$ & (b) \\
\hline & & & & & & & & & & & & & & 1 & $\cdot \mid q \cdot r^{m-m}$ & $\cdot 1099^{\circ *}$ & $\cdot 109 \cdot^{\cdots *}$ & $\cdot \mid k f f^{\infty n}$ & $-\cdot / N A F$ & (9) \\
\hline & & & & & & & & & & & & & 1 & $\cdot \mid q \cdot r^{*-*}$ & $\cdot \mid \Delta \wedge \Delta^{* \pi}$ &.$/ \pi 19$ & $\cdot / r \Delta q$ & $\cdot / r \Delta$. &.$- / 11 r$ & (v) \\
\hline & & & & & & & & & & & & 1 &.$|q T|^{\cdots}$ & $\cdot|V| \cdots \mid \cdot$ & "םrז/. &.$/ T \vee \varphi^{\prime \prime}$ & $\cdot / D F A$ & $\cdot / 4 \Delta \cdot \cdots$ &.$/ . t$. & (^) \\
\hline & & & & & & & & & & & 1 & $\cdot / 9 \cdot f^{n=}$ &.$/ 4 \varepsilon^{* *}$ & $\cdot / \Delta \& q^{* \pi}$ &.$/$ rr. & . /rsq & $\cdot / \Delta V V^{-m}$ & . &.$/ T I K$ & (9) \\
\hline & & & & & & & & & & 1 & . & $\cdot \mid r \lambda e^{n-}$ & $\cdot / r \Lambda \cdot{ }^{*}$ & $\cdot / V \cdot r^{n+r}$ & $\cdot / 0 \cdot e^{n-n}$ & . & $\cdot / r \Delta \cdot$ & . & $-\cdot / 1 \wedge \Lambda$ & $(1 \cdot)$ \\
\hline & & & & & & & & & 1 & $\cdot \mid \Delta \Delta \Delta^{m-\sigma}$ & . Irs & - MFF &. $\mid r 81$ &.$/ A r V^{n-m}$ & $\cdot / r \mid \Lambda$ & $\cdot|q Y|^{\circ}$ & . KG4 & .1 .91 & . H FT & (11) \\
\hline & & & & & & & & 1 &. $\mid F \& Y^{\prime \prime}$ & $\cdot \mid f \Delta f^{n-\pi}$ & .1 .94 & - Kra &.$/ .14$ & . IKTF &.$/ .1$ &. $\mid\left(\left.49\right|^{\pi}\right.$ &.$/ 1 \wedge 9$ & .1 .99 &.$- / 19 V$ & (IT) \\
\hline & & & & & & & 1 & $\cdot / \Lambda \Delta \cdot{ }^{* \uparrow}$ & $\cdot / r \Delta q^{*}$ &. $\mid F r \Lambda^{n *}$ & $.1 \cdot 19$ & . / $\wedge \Delta$ &.$/ \cdot T V$ & . TVY &.$/ 1 F$. & $. \pi / r$ & $\cdot / \cdot \Delta r$ & .1 .91 & $-\cdot / 1 \cdot 1$ & (Ir) \\
\hline & & & & & & 11 & $\cdot / r \wedge \varepsilon^{*}$ & . ATrT &.$/ 4 \wedge 9$ &.$/ 94 q^{* *}$ &.$/ \% \wedge q^{*}$ & $\cdot|\Delta r|^{* *}$ & . RTA & $\cdot \mid q \cdot f^{7 m}$ & . & $\cdot / r \Delta r^{*}$ & $\cdot / 4 \Delta r^{m-*}$ & . &.$- \mid 191$ & (lf) \\
\hline & & & & & 1 &.$|r \&|^{\circ}$ &.$/ I V$ & $\cdot / r V \wedge$ & $\cdot / 099^{m *}$ & $\cdot / 4 \wedge \cdot{ }^{*-m}$ & $\cdot|r \Delta|^{\pi}$ & $\cdot \mid \Delta \cdot 1^{* \cdots}$ & $\left.\cdot|q|\right|^{\cdots m}$ & $\cdot|9 \Delta|^{-\pi}$ & $\cdot / T \wedge \Delta$ & $\cdot \mid \Delta \& V^{m *}$ & $\cdot \mid \Delta 99^{\circ *}$ & $\cdot / T V \Delta$ & $-/ 1 / \vee q$ & $(\mid \Delta)$ \\
\hline & & & & 1 & $\cdot / 4 \wedge 9^{m *}$ & $\cdot \cdot \mid q \cdot 1^{m \pi}$ & $-\cdot / l \Delta F$ & -.1 .91 &.$\pi r$. & $-/ / f q$ & -.1 .91 & $-\cdot / \cdot v$ & . / & $-\cdot / \cdot T_{F}$ & $.1 . \mu f$ & $\cdot 11 \cdot 4$ & $\cdot / \cdot \Delta$ & $-\cdot / 1 \cdot f$ & $-\cdot \cdot r_{\mid}$ & (19) \\
\hline & & & 1 & $\cdot / r \Delta S$ & $\cdot / M M N$ & Mff" & $\cdot 11 \cdot f$ & . & $\cdot \mid \$ 49^{\prime \prime-}$ &.$|48|^{\prime \prime}$ & - raq & $\cdot / r \Delta \Delta^{*}$ & . MAT" & $\cdot \mid \Delta \Delta r^{N-m}$ & ./Ir & $\cdot / \Delta V V^{-\infty}$ & . $/ \Delta \mathbf{A T}^{-\pi}$ &.$/ 4 \wedge q^{*}$ & - & (IV) \\
\hline & & 1 & - - KFA & $-\cdot / r q r^{*}$ & $-\cdot 19 \Delta r^{* * *}$ &.$- / 4 Y q$ & - $/ / Q T$ & -.1190 & $\underset{* *}{-}<\notin q q$ & $-\cdot \mid \kappa r \cdot{ }^{* *}$ & $-\cdot|| \Delta \mid$ & $-\cdot / r T V$ & $-\cdot / \Delta \cdot r^{* *}$ & $-\cdot / \uparrow q \varphi^{* *}$ & $\cdot / \mu *^{*}$ & $-\cdot / r \Lambda \Lambda$ & $\cdot / r \wedge \vee$ &.$/ 19$. & -.1 .19 & $(1 \Lambda)$ \\
\hline & 1 & $\cdot|V \psi|^{-*}$ & . KGY & . $/ \Delta \mid T^{m * n}$ & $\cdot|\Lambda \Delta|^{-\pi}$ & $\cdot / r 19$ & זrון. & . /Nイ & $\cdot \mid q V r^{m *}$ &.$|r q|^{*}$ & - raA & . $/ 4 F D^{*=}$ & $\cdot / Q T \Lambda^{N *}$ &.$/ 499^{* * *}$ & - r raf &.$/\left.49\right|^{\circ}$ & - / TTa &.$/ .94$ & .1 .11 & (19) \\
\hline 1 & $\cdot \mid q \cdot r^{m *}$ & $-\| \Delta T \Lambda^{* *}$ & $\cdot|f \Delta|^{-n}$ & _./IrV & $.19 \Delta \Lambda^{N "}$ & $\cdot / V I r^{*-\pi}$ & . &.$/ 191$ & $\cdot / F F D^{* n}$ &.$/ q \mu F^{m-\pi}$ & . & . /GYT & $\cdot / F V r^{m *}$ & $\cdot / V \Delta V^{*-*}$ &.$/ 49 \Delta^{\circ}$ & $\cdot / r \wedge \varphi^{\pi}$ & $\cdot \mid \Delta V V^{-m}$ & . $/$ TQT & $\cdot / \cdot v G$ & $(T \cdot)$ \\
\hline
\end{tabular}




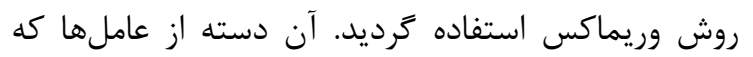

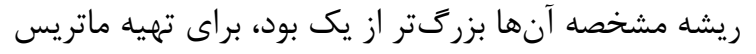

ضرايب عاملى انتخاب شدند (جداول هـ هو و و).

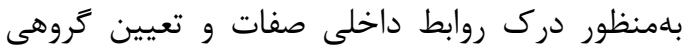

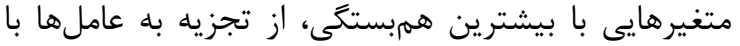

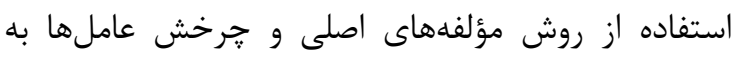

\begin{tabular}{|c|c|c|c|}
\hline درصد تجمعى واريانس & واريانس نسبى & مقادير ويزه عاملها & ماملها \\
\hline rI/^94 & rI/19q & $f / r v q$ & 1 \\
\hline$f \cdot|0| 9$ & $|N / 9 Y|$ & T/VTF & r \\
\hline$\Delta \varphi / / \wedge q$ & $10 / 9 V T$ & DוT & r \\
\hline $9 V / 994$ & $11 / \Delta \cdot \Delta$ & $r / r \cdot 1$ & i \\
\hline VN/qKq & $11 / r \Delta \varphi$ & $r / T \Delta 1$ & $a$ \\
\hline
\end{tabular}

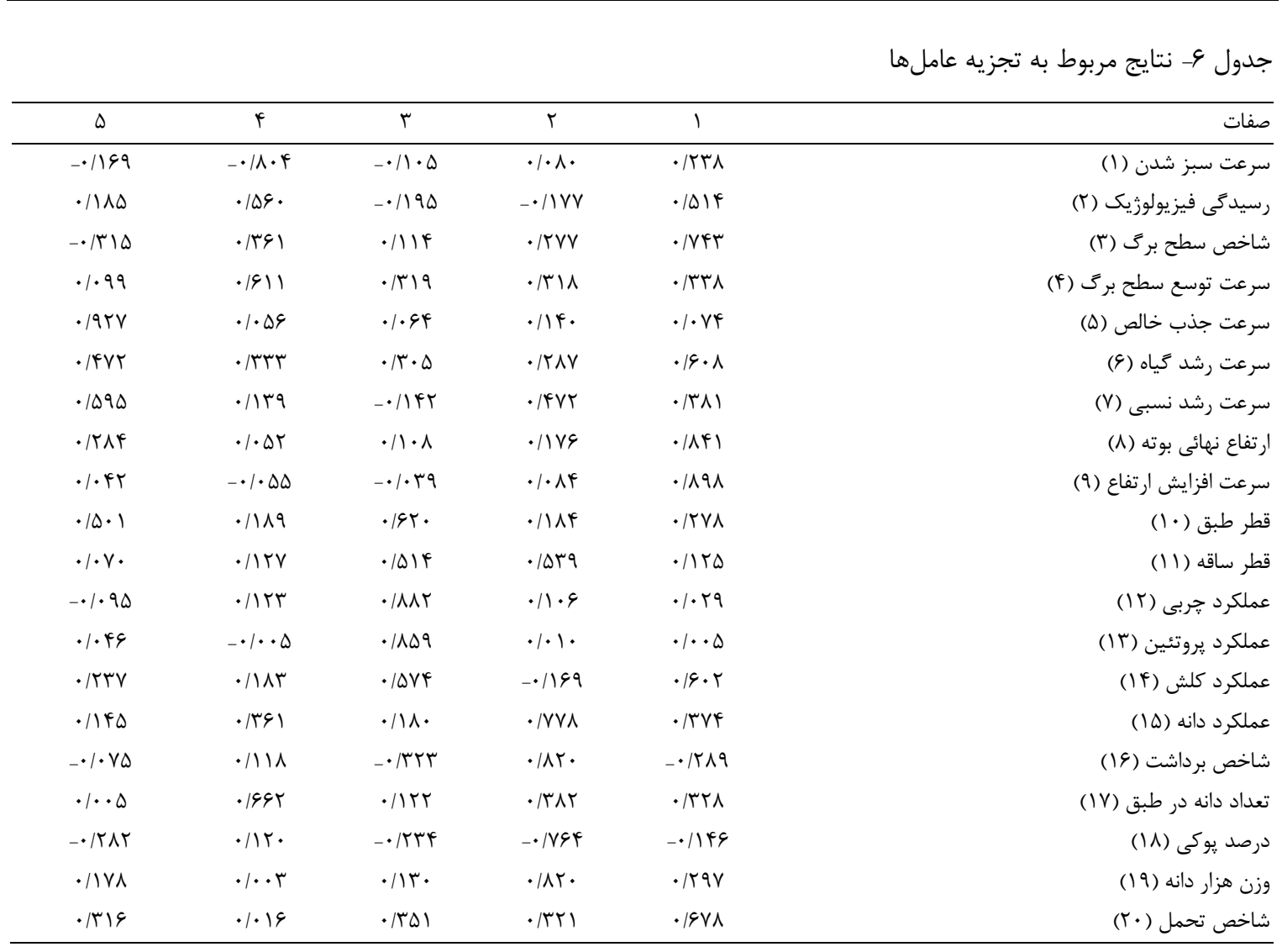

وزن هزار دانه ضريب عاملى مثبت و براى صفاتى جون

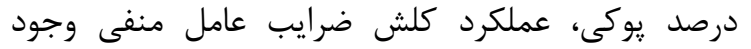
داشت كه اين عامل نيز عامل عملكرد كمى نام كرفت

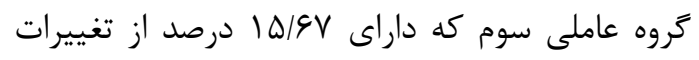

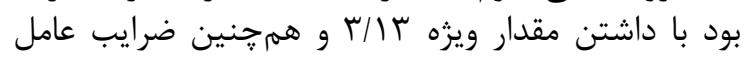

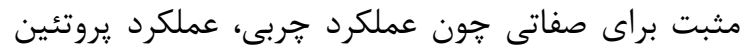
به صورت عامل عملكرد كيفى نامخذارى شد شدئ

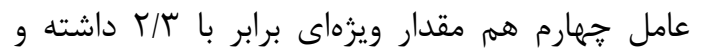

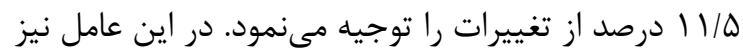

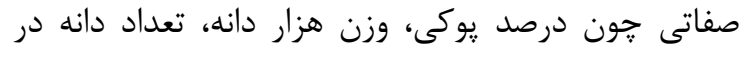

در اين تجزيه ينج عامل به دست آمد كه مجموعاً

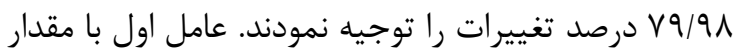

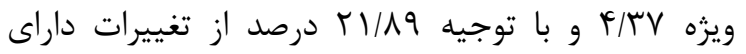

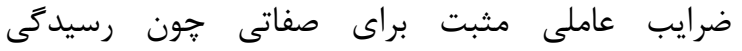

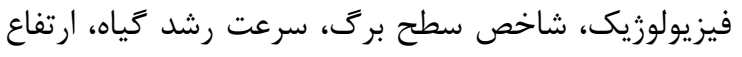

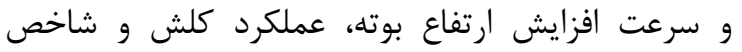

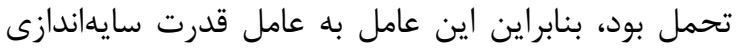

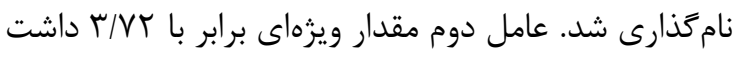

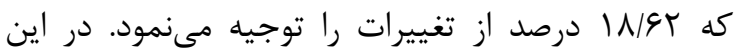

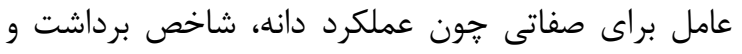


ارتفاع، سرعت جذب خالص، سرعت رشد نسبى، سرعت

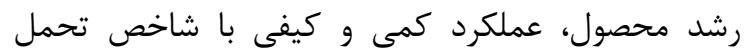

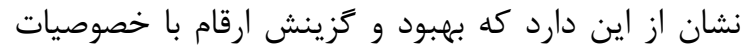

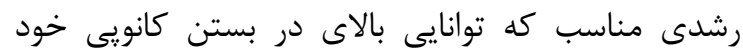

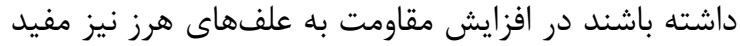

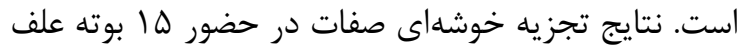

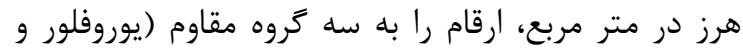

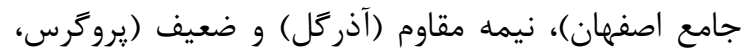

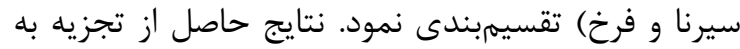

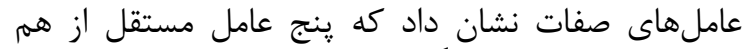

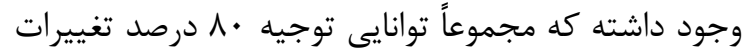
رارند.

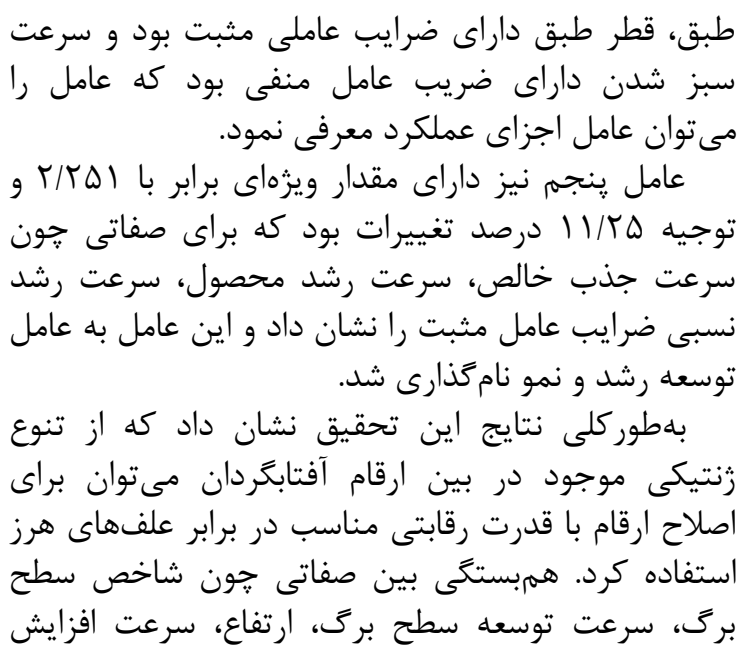

منابع

1. Balolic, I., J. Crnobarac and N. Dusanic. 2007. Planting data effect on oil yield in sunflower. Helia, 47: 153-158.

2. Bertholdsson, N.O. 2004. Variation in allelopathic activity over one hundred years of barley selection and breeding. Weed Research, 44: 78-86.

3. Bertholdsson, N.O. and R. Jönsson. 1994. Weed competition in barley and oats. In: Proc. 3rd ESA Congress. Abano-Padova, 656-657.

4. Bertholdsson, N.O. 2005. Early vigour and allelopathy: two useful traits for enhanced barley and wheat competitiveness against weeds. Weed Research, 45: 94-102.

5. Caton, B.P., T.C. Foin, J.C. Hill and A.M. Mortimer. 2001. Measuring crop competitiveness and indentiying associated traits in cultivar field trials. Eighteenth Asian Pacific weed Sciences. Soc Conf., Beijing, China, 139-145 pp.

6. Davies, D.K.H., S.P. Hoad, P.R. Maskell and K. Topp. Looking at cereal varieties to help reduce weed control inputs. Proceedings Crop Protection Northern Britain, 159 -163 pp.

7. Didon, U.M.E. 2002. Growth and development of barley cultivars in relation to weed competition. Thesis, Swedish University of Agricultural Sciences, Uppsala, Acta Universitatis Agriculture Sueciae Agraria, $332 \mathrm{pp}$.

8. Didon, U.M.E. and M.L. Hansson. 2002. Competition between six spring barley (Hordeum vulgare ssp. vulgare L.) cultivars and two weed flora in relation to interception of photosynthetic active radiation. Biological Agriculture \& Horticulture, 20: 257-274.

9. Didon, U.M.E. and U. Boström. 2003. Growth and development of six barley (Hordeum vulgare ssp. vulgare L.) cultivars in response to a model weed (Sinapis alba L.). Journal of Agronomy and Crop Science, 189: 409-417.

10.Donovan, J., K.N. Harker, G.W. Claytion and L.M. Hall. 2000. Wild Oat (Avena fatua) Interference in Barley (Hordeum vulgare) is influenced by Barley Variety and Seeding Rate1. Weed Technology, 14: 624-629.

11.FAO. 2012. Preliminary 2012 Data Now Available. http://www.fao.org. 25 September 2013.

12.Faustini, F. and R. Paolini. 2005. Organically grown durum wheat (Triticum durum Desf.) varieties under different intensity and time of mechanical weed control. Proc. 13th EWRS Symposium, Bari (IT), 20-23 June, CD-ISBN 90-809789-1-4.

13.Hoad, S., D.H.K. Davies and C.F.E. Topp. 2006. Designing crops for low input and organic sysyems: enhancing wheat competitive ability against weeds. Proc. Crop Protection in Northern Britain, 157-162.

14.Kruepl, C., S. Hoad, K. Davies, N.O. Bertholdsson and R. Paolini. 2007. Weed Competitiveness. Susvar Handbook, 17 pp.

15. Mirshekari, B. 2011. Sunflower yield and harvest index in monoculture and in competition with Amaranthus. Journal of modern science of sustainable agriculture, 6: 73-88. 


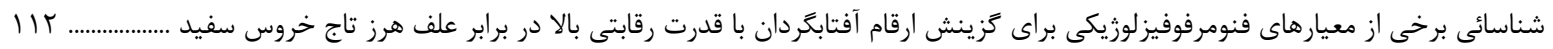

16.Mokhtari, S., N.W. Galwey, R.D. Cousens and N. Thurling. 2002. The genetic basis of variation among wheat $\mathrm{F} 3$ genotypes in tolerance to competition by ryegrass (Lolium rigidum). Euphytica, 124: 355-364.

17.Ngouajio, M., M.E. Mc-Giffen and K.J. Hembree. 2001. Tolerance of tomato cultivar to velvetleaf interference. Weed Science, 49: 91-98.

18.Nunes, U.R., A.A. Silva, M.S. Reis, C.S. Sediyama and T. Sediyama. 2003. Soybean seed osmoconditioning effect on the crop competitive ability against weeds. Planta Daninha, 21: 2735.

19.So, Y.F., M.M. Williams, J.K. Pataky and A.S. Davis. 2009. Principal canopy factors of sweet corn and relationships to competitive ability with wild-proso millet (Panicum miliaceum). Weed Science. 57: 296-303.

20.Tepe, I., M. Erman, A. Yazlik, R. Levent and K. Ipek. 2005. Comparison of some winter lentil cultivars in weed-crop competition. Crop Protection, 24: 585-589.

21.Traore, S., S.C. Mason, A.R. Martin, D.A. Mortensen and J.J. Spotanski. 2003. Velvetleaf Interference Effects on Yield and Growth of Grain Sorghum. Agronomy Journal, 95: 1602-1607.

22.Wicks, G.A., A.T. Nordquist, P.S. Baenziger, R.N. Klein, R.H. Hammons and J.E. Watkins. 2004. Winter Wheat Cultivar Characteristics Affect Annual Weed Suppression1 Weed Technology, 18: 988-998.

23.Williams, M.M., R.A. Boydston and A.S. Davis. 2007. Wild proso millet (Panicum miliaceum) suppressive ability among three sweet corn hybrids. Weed Science, 55: 245-251.

24.Wilson, J.B. 1988. Shoot competition and root competition. Journal of Applied Ecology, 25: 279-96.

25.Zand, E. and H.J. Beckie. 2002. Competitive ability of hybrid and open pollinated canola (Brassica napus L.) with wild oat (Avena fatua L.). Canadian Journal Plant Science, 82: 473480 . 


\title{
Identification of Some Pheno-Morpho-Physiological Measures for the Selection of Highly Competitive Sunflower (Helianthus annuus L.) Varieties Against Tumble Pigweed (Amaranthus albus L.) using Multivariate Statistical Methods
}

\author{
Mohammad Javad Babaie Zarch ${ }^{1}$, Sohrab Mahmoodi ${ }^{2}$ and Seyyed Vahid Eslami ${ }^{2}$ \\ 1- Graduated M.Sc. Student, University of Birjand, (Corresponding author: javadbabaei67@gmail.com) \\ 2- Associate Professor, University of Birjand \\ Received: January 27, $2014 \quad$ Accepted: May 12, 2014
}

\begin{abstract}
Using the genetic diversity of crop cultivars to improve crop resistance against various biotic and abiotic stresses is effective way to develop sustainable agriculture. Therefore, aimed to investigate the effect of cultivar type as well as introduce the most important traits related to competitive ability of sunflower cultivars against tumble pigweed, a factorial experiment with three replications was at the agricultural research station, University of Birjand during 2012. Experimental treatment included six cultivars of oily sunflower (Azargol, Syrna, Farukh, Jame Isfahan, Progress and Euroflor) and two different densities of tumble pigweed (zero and $15 \mathrm{plant} / \mathrm{m}^{2}$ ). ANOVA results showed that there was a remarkable genetical diversity among studied cultivars and significant reductions in studied traits values observed where tumble pigweed was present. Moreover, there were significant positive correlations between traits such as leaf area index, plant height, the rate of leaf area and height development, relative growth rate, seed yield, etc. with the tolerance index. Factor analysis also identified 5 factor groups which justified $79 \%$ of the variations. Overall, Jame Isfahan showed the greatest competitive ability against tumble pigweed compared with other studied cultivars.
\end{abstract}

Keywords: Competitive Ability, Jame Isfahan, Leaf Area Index, Sustainable Agriculture, Tolerance Index 